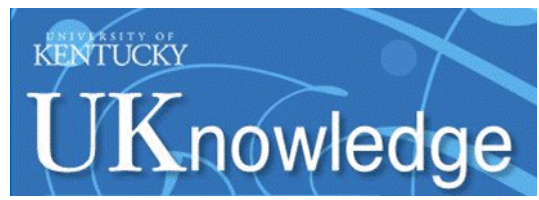

University of Kentucky

UKnowledge

$5-27-2018$

\title{
Fracture Initiation in a Transversely Isotropic Solid: Transient Three Dimensional Analysis
}

Louis Milton Brock

University of Kentucky, louis.brock@uky.edu

Follow this and additional works at: https://uknowledge.uky.edu/me_facpub

Part of the Mechanical Engineering Commons, and the Structures and Materials Commons Right click to open a feedback form in a new tab to let us know how this document benefits you.

\section{Repository Citation}

Brock, Louis Milton, "Fracture Initiation in a Transversely Isotropic Solid: Transient Three Dimensional Analysis" (2018). Mechanical Engineering Faculty Publications. 44.

https://uknowledge.uky.edu/me_facpub/44

This Article is brought to you for free and open access by the Mechanical Engineering at UKnowledge. It has been accepted for inclusion in Mechanical Engineering Faculty Publications by an authorized administrator of UKnowledge. For more information, please contact UKnowledge@lsv.uky.edu. 
Fracture Initiation in a Transversely Isotropic Solid: Transient Three Dimensional Analysis

Digital Object Identifier (DOI)

https://doi.org/10.2140/jomms.2018.13.171

Notes/Citation Information

Published in Journal of Mechanics of Materials and Structures, v. 13, no. 2, p. 171-190.

The publisher has granted the permission for posting the article here. 


\title{
FRACTURE INITIATION IN A TRANSVERSELY ISOTROPIC SOLID: TRANSIENT THREE DIMENSIONAL ANALYSIS
}

\author{
LOUIS M. BROCK
}

\begin{abstract}
A transversely isotropic solid is at rest, and contains a semi-infinite, plane crack. The axis of rotational material symmetry lies in the crack plane. Application of normal point forces to each face of the crack causes transient 3D growth. The related problem of discontinuities in displacement and traction that exist on regions that exhibit dynamic similarity is first considered. Analytic results are obtained in integral transform space. These lead to equations of the Wiener-Hopf type for the fracture problem. Analytic solutions are again obtained and, upon inversion, subjected to a dynamic energy release rate criterion that includes kinetic energy. A particular form of rapid growth in time of the forces is found to cause crack growth rates that indeed vary with position, but not with time. The influence of anisotropy upon wave speeds and crack edge contour are examined.
\end{abstract}

\section{Introduction}

Fracture initiation caused by mixed-mode, point-force loading at the edge of a semi-infinite plane crack is considered in [Brock 2017a; 2017b]. Analysis in each case is 3D and transient. The crack exists in an unbounded, isotropic, and isothermal solid in [Brock 2017b], while the solid is thermoelastic and initially at uniform (absolute) temperature in [Brock 2017a]. The dynamic energy release rate criterion [Freund 1972; 1990] is imposed, but with kinetic energy taken into account [Gdoutos 2005]. In [Brock $2017 \mathrm{~b}$ ] it is found that a particular time history for the loading can generate a crack edge contour that is dynamically similar; i.e., its shape is time-invariant. The same result is found in [Brock 2017a] for the fracture initiation phase. In both studies, inclusion of kinetic energy enhances the (constant) rate at which the crack expands into the material. In both studies the expanding contour is semicircular only when shearing forces are absent.

This study concerns the effect of anisotropy on the fracture process. The solid is isothermal, so that the time history considered in [Brock 2017a; 2017b] again predicts a dynamically similar crack contour. However, the solution results will be considered in the context of a (perhaps brief) fracture initiation phase. Moreover, to emphasize the effects of transverse isotropy, only normal point force loading is considered. In this regard, when the axis of material rotational symmetry is perpendicular to a plane:

(1) Plane strain problems are governed by the two elastic constants associated with the plane, i.e., are effectively isotropic [Scott and Miklowitz 1967].

(2) Some results for 3D problems are independent of direction in the plane [Brock 2013].

Keywords: transverse isotropy, transient, fracture initiation, kinetic energy, crack contour. 
Here therefore the material rotational symmetry axis lies in the crack plane. Moreover, the initial crack edge is not aligned with a principal axis in the plane. The solution process follows closely that employed for [Brock 2017a; 2017b]. Process steps are clearly identified, but resulting formulas are generally confined to those unique to the problem considered here.

\section{Problem statement}

Consider an unbounded, transversely isotropic solid with principal axes defined by fixed Cartesian basis $\boldsymbol{x}_{0}=\boldsymbol{x}_{0}\left(x_{k}^{0}\right), k=(1,2,3)$. A closed crack $A_{C}\left(x_{3}^{0}, \xi_{1}^{0}<0\right)$ with boundary $C\left(\xi_{1}^{0}, x_{3}^{0}\right)=0$ is at rest for time $t \leq 0$, where

$$
\left[\begin{array}{l}
\xi_{1}^{0} \\
\xi_{2}^{0}
\end{array}\right]=\left[\begin{array}{rr}
\cos \theta & \sin \theta \\
-\sin \theta & \cos \theta
\end{array}\right]\left[\begin{array}{l}
x_{1}^{0} \\
x_{2}^{0}
\end{array}\right] \quad(|\theta|<\pi / 2) .
$$

It is noted that the axis of material rotational symmetry is parallel to the $x_{2}^{0}$-axis. For $t>0$ compressive point forces appear on both crack faces at $\left(x_{1}^{0}=0-, x_{2}^{0}=0, x_{3}^{0}=0 \pm\right)$. Brittle fracture is instantaneous, and the crack extends outward from $x_{0}=0$ in the positive $\xi_{1}^{0}$-direction. The crack now occupies region $A_{C}+\delta A$ and boundary $C$ includes a concave bulge:

$$
\begin{gathered}
\sqrt{\left(x_{1}^{0}\right)^{2}+\left(x_{2}^{0}\right)^{2}}=l(\theta, \psi, t), \quad l(\theta, \psi, t)=V(\theta, \psi) t, \\
0<V<V_{R}, \quad \psi=\tan ^{-1} \frac{\xi_{2}^{0}}{\xi_{1}^{0}} \quad(|\psi|<\pi / 2) .
\end{gathered}
$$

Introduction of an orientation angle $\psi$ with respect to coordinates $\left(\xi_{1}^{0}, \xi_{2}^{0}\right)$ proves useful in the derivation of solutions. Equation (2) implies a dynamically similar process, and requires that (speed parameter) $V$ is subsonic and below the Rayleigh value $V_{R}$. Displacement $\boldsymbol{u}\left(u_{k}\right)$ and traction $\boldsymbol{T}\left(\sigma_{i k}\right)$ are field variables. If body forces are neglected [Payton 1983; Jones 1999],

$$
\begin{gathered}
\nabla \cdot \boldsymbol{T}-\rho D^{2} \boldsymbol{u}=0, \\
{\left[\begin{array}{l}
\sigma_{11} \\
\sigma_{22} \\
\sigma_{33}
\end{array}\right]=\left[\begin{array}{lll}
C_{11} & C_{12} & C_{13} \\
C_{12} & C_{22} & C_{12} \\
C_{13} & C_{12} & C_{33}
\end{array}\right]\left[\begin{array}{l}
\partial_{1} u_{1} \\
\partial_{2} u_{2} \\
\partial_{3} u_{3}
\end{array}\right],} \\
\sigma_{2 k}=C_{44}\left(\partial_{2} u_{k}+\partial_{k} u_{2}\right), \quad k=(1,3), \quad \sigma_{31}=C_{55}\left(\partial_{3} u_{1}+\partial_{1} u_{3}\right) .
\end{gathered}
$$

Components $\left(u_{k}, \sigma_{i k}\right)$ are functions of $\left(x_{0}, t\right), \partial_{k} f=\partial f / \partial x_{k}$ and $\left(\nabla, \nabla^{2}, 1\right)$ respectively are gradient and Laplacian operators and identity tensor. Here $(D f, \dot{f})$ signify time differentiation in basis $x_{0}$ and $\left(C_{i k}, \rho\right)$ are the elastic constants and mass density, and $C_{13}=C_{11}-2 C_{55}$. Here reference quantities are shear modulus and shear wave speed:

$$
\mu=C_{44}, \quad V_{4}=\sqrt{C_{44} / \rho} .
$$

These quantities give dimensionless parameters

$$
c=\frac{V}{V_{4}}, \quad d_{1}=\frac{C_{11}}{C_{44}}, \quad d_{2}=\frac{C_{22}}{C_{44}}, \quad d_{5}=\frac{C_{55}}{C_{44}}, \quad d_{12}=\frac{C_{12}}{C_{44}}, \quad d_{13}=\frac{C_{13}}{C_{44}}=d_{1}-2 d_{5} .
$$


For $x_{3}^{0}=0 \pm,\left(x_{1}^{0}, x_{2}^{0}\right) \in A_{C}+\delta A(t>0)$,

$$
\sigma_{31}=\sigma_{32}=0, \quad \sigma_{33}=-P \delta\left(x_{1}^{0}\right) \delta\left(x_{2}^{0}\right) .
$$

For $x_{3}^{0}=0,\left(x_{1}^{0}, x_{2}^{0}\right) \notin A_{C}+\delta A(t>0)$,

$$
\left[u_{k}\right]=0 .
$$

In (5) force $P$ is a positive constant, $\delta(f)$ denotes Dirac function, and $[f]=f^{(+)}-f^{(-)}$, where $f^{( \pm)}=f\left(\xi_{1}^{0}, \xi_{2}^{0}, 0 \pm, t\right)$. In addition $\left[u_{k}\right]$ must vanish continuously on $C$, but $\sigma_{3 k}$ may exhibit (integrable) singular behavior on $C$. For $t \leq 0,(\boldsymbol{u}, \boldsymbol{T}) \equiv 0$, and for finite $t>0,(\boldsymbol{u}, \boldsymbol{T})$ must be bounded as $\left|x_{0}\right| \rightarrow \infty$.

\section{Discontinuity problem}

A common practice for solving crack problems is to represent the relative motion of crack faces as unknown discontinuities in displacement; see, e.g., [Barber 1992]. To implement that procedure, the related problem of discontinuities in $\left(u_{k}, \sigma_{3 k}\right)$ is now considered: The unbounded solid is again at rest when for time $t>0$ the discontinuities are imposed in the same region $A_{C}+\delta A$ of the $x_{1}^{0} x_{2}^{0}$-plane. In place of (5) we have for $x_{3}^{0}=0,\left(x_{1}^{0}, x_{2}^{0}\right) \in A_{C}+\delta A(t>0)$

$$
\left[u_{k}\right]=\Delta_{k}, \quad\left[\sigma_{3 k}\right]=\Sigma_{k} .
$$

For $x_{3}^{0}=0,\left(x_{1}^{0}, x_{2}^{0}\right) \notin A_{C}+\delta A(t>0)$,

$$
\left[u_{k}\right]=\left[\sigma_{3 k}\right]=0 .
$$

Here $\left(\Delta_{k}, \Sigma_{k}\right)$ are continuous functions of $\left(x_{1}^{0}, x_{2}^{0}, t\right)$. They vanish on $C$ and for $t \leq 0$ are bounded in $A_{C}+\delta A$ for $\sqrt{\left(x_{1}^{0}\right)^{2}+\left(x_{2}^{0}\right)^{2}} \rightarrow 0$. Therefore, as in the crack problem, $(\boldsymbol{u}, \boldsymbol{T}) \equiv 0$ for $t \leq 0$, and are bounded as $|\boldsymbol{x}| \rightarrow \infty$ for finite $t>0$.

\section{Transform solution}

An effective procedure (see, e.g., [Brock and Achenbach 1973]) for 2D transient study of semi-infinite crack extension at constant speed employs:

(1) Coordinates that translate with the crack edge.

(2) Unilateral temporal and bilateral spatial integral transform [Sneddon 1972].

In view of (1) a translating basis $\boldsymbol{x}$ is defined for $|\psi|<\pi / 2$ as

$$
\begin{gathered}
\xi_{1}=\xi_{1}^{0}-[c(\theta, \psi) \cos \psi] s, \quad \xi_{2}=\xi_{2}^{0}-[c(\theta, \psi) \sin \psi] s, \quad x_{3}=x_{3}^{0}, \\
s=V_{4} t, \quad c(\theta, \psi)=\frac{V(\theta, \psi)}{V_{4}}, \\
D f=\dot{f}=V_{4}\left[\partial-c(\psi)\left(\partial_{1} f \cos \psi+\partial_{2} f \sin \psi\right)\right], \\
\partial=\frac{\partial f}{\partial s}, \quad \partial_{k} f=\frac{\partial f}{\partial x_{k}^{0}}, \quad k=(1,2) .
\end{gathered}
$$


The temporal Laplace transform operation is

$$
L(f)=\hat{f}=\int f(s) \exp (-p s) d s .
$$

Integration is over positive real $s$ and $\operatorname{Re}(p)>0$. A double spatial integral transform and inversion, respectively, can be defined [Sneddon 1972] by

$$
\begin{aligned}
& \tilde{f}\left(p, q_{1}, q_{2}\right)=\iint \hat{f}\left(p, x_{1}, x_{2}\right) \exp \left[-p\left(q_{1} x_{1}+q_{2} x_{2}\right)\right] d x_{1} d x_{2}, \\
& \hat{f}\left(p, x_{1}, x_{2}\right)=\left(\frac{p}{2 \pi i}\right)^{2} \iint \tilde{f}\left(p, q_{1}, q_{2}\right) \exp \left[p\left(q_{1} x_{1}+q_{2} x_{2}\right)\right] d q_{1} d q_{2} .
\end{aligned}
$$

In light of (1) and (7a),

$$
x_{1}=x_{1}^{0}-[c(\theta, \psi) \cos \chi] s, \quad x_{2}=x_{2}^{0}-[c(\theta, \psi) \cos \chi] s, \quad \chi=\theta+\psi .
$$

Integration in $(9 \mathrm{a})$ is over $\left(x_{1}, x_{2}\right)$; integration in $(9 \mathrm{~b})$ is along the imaginary $\left(q_{1}, q_{2}\right)$-axes. It is noted that $(\boldsymbol{x}, s)$ have dimensions of length, $p$ has dimensions of inverse length, and $\left(q_{1}, q_{2}\right)$ are dimensionless. Because (1) involves a speed that varies with direction, application of (8) and (9a) to (3), (4), and (6) is complicated. Despite use of $(\theta, \psi)$ the discontinuity problem is not axially symmetric. However, 3D studies of sliding and rolling contact [Brock 2012] and crack growth [Brock 2017a; 2017b] suggest transformations

$$
\begin{gathered}
\operatorname{Im}\left(q_{1}\right)=\operatorname{Im}(q) \cos \chi, \quad \operatorname{Im}\left(q_{2}\right)=\operatorname{Im}(q) \cos \chi \\
x_{1}=\xi \cos \chi, \quad x_{2}=\xi \sin \chi, \quad \xi=\sqrt{x_{1}^{2}+x_{2}^{2}}=\sqrt{\xi_{1}^{2}+\xi_{2}^{2}} .
\end{gathered}
$$

Here $\operatorname{Re}(q)=0+,|\operatorname{Im}(q)|,|\xi|<\infty,|\psi|<\pi / 2$, and parameters $(\xi, \chi)$ and $(q, \chi)$ resemble quasipolar coordinates, i.e.,

$$
d x_{1} d x_{2}=|\xi| d \xi d \chi, \quad d q_{1} d q_{2}=|q| d q d \chi
$$

In particular the portion of crack contour $C$ that bounds newly created crack surface $\delta A$ can be defined as $(\xi=0,|\psi|<\pi / 2)$. The uncoupling effect of (10) leads to the combination

$$
\begin{gathered}
\tilde{f}\left(p, q_{1}, q_{2}\right) \rightarrow \bar{f}(p, q, \chi), \\
\hat{f}(p, \xi, \chi)=-\frac{p^{2}}{2 \pi} \int \frac{|q|}{q} \bar{f}(p, q, \chi) \exp (p q \xi) d q .
\end{gathered}
$$

Integration is along the positive $(\operatorname{Re}(q)=0+)$ side of the $\operatorname{Im}(q)$-axis.

In view of (8)-(11) and (11a), Equations (3), (4), and (6) give a corresponding set in transform space by making formal substitutions

$$
\begin{gathered}
\nabla \rightarrow\left(p q \cos \chi, p q \sin \chi, \partial_{3}\right), \quad D \rightarrow \frac{p}{V_{4}} \beta, \quad \nabla^{2} \rightarrow \partial_{3}^{2}+p^{2} q^{2} \\
\beta=1-c q .
\end{gathered}
$$


Set elements that correspond to (3a) are homogeneous, ordinary differential equations in $x_{3}$, with characteristic functions $p A_{5}(q)$ and $p A_{ \pm}(q)$ :

$$
\begin{gathered}
A_{5}(q)=\frac{1}{\sqrt{d_{5}}} \sqrt{\beta^{2}-q^{2} c_{5}^{2}}, \quad c_{5}=\sqrt{d_{5} \cos ^{2} \chi+\sin ^{2} \chi}, \\
A_{ \pm}(q)=\sqrt{-1 /\left(4 d_{1}\right)\left(\Omega_{+} \pm \Omega_{-}\right)^{2}}-q^{2} \cos ^{2} \chi \\
\Omega_{ \pm}=\sqrt{\left(\sqrt{d_{1} C_{2}} \pm \sqrt{C_{0}}\right)^{2}-m^{2} q^{2} \sin ^{2} \chi} \\
C_{0}=q^{2} \sin ^{2} \chi-\beta^{2}, \quad C_{2}=d_{2} q^{2} \sin ^{2} \chi-\beta^{2}, \quad m=1+d_{12} .
\end{gathered}
$$

It is noted that (14a) and (14b) yield the convenient result

$$
d_{1}\left(A_{-}^{2}-A_{+}^{2}\right)=\Omega_{+} \Omega_{-} .
$$

The branch point parameter $c_{5}$ for $A_{5}$ is a dimensionless shear wave speed. Similarly $A_{ \pm}$respectively are associated with dimensionless wave speeds $c_{ \pm}$:

$$
\begin{gathered}
c_{ \pm}=\frac{1}{2}\left(C_{ \pm} \pm C_{-}\right) \\
C_{ \pm}=\sqrt{1+d_{1} \cos ^{2} \chi+d_{2} \sin ^{2} \chi \pm 2 \sqrt{d_{1} \cos ^{4} \chi+d_{2} \sin ^{4} \chi+\Gamma \sin ^{2} \chi \cos ^{2} \chi}} \\
\Gamma=1+d_{1} d_{2}-m^{2}
\end{gathered}
$$

It is noted that

$$
A_{+} A_{-}=\frac{1}{\sqrt{d_{1}}} \sqrt{\beta^{2}-q^{2} c_{+}^{2}} \sqrt{\beta^{2}-q^{2} c_{-}^{2}} .
$$

In [Payton 1983] parameter combinations $(m, \Gamma)$ are used to characterize transversely isotropic materials; the characterization scheme is summarized in Appendix A. Results in what follows are often general. However, some key expressions and associated calculations will be illustrated in terms of the Category 1 material. In light of (13)-(15), the corresponding equations give displacement transform $\bar{u}=\bar{u}_{5}+\bar{u}_{+}+\bar{u}_{-}$ where components are

$$
\begin{gathered}
{\left[\begin{array}{l}
\left(\bar{u}_{5}\right)_{1} \\
\left(\bar{u}_{5}\right)_{2} \\
\left(\bar{u}_{5}\right)_{3}
\end{array}\right]=\left[\begin{array}{c}
( \pm) A_{5} \\
0 \\
q \cos \chi
\end{array}\right] U_{5}^{( \pm)} \exp \left(-p A_{5}\left|x_{3}\right|\right)} \\
{\left[\begin{array}{c}
\left(\bar{u}_{ \pm}\right)_{1} \\
\left(\bar{u}_{ \pm}\right)_{2} \\
\left(\bar{u}_{ \pm}\right)_{3}
\end{array}\right]=\left[\begin{array}{c}
(\mp) m^{2} q \sin \chi \cos \chi \\
( \pm) Q_{ \pm} \\
m q A_{ \pm} \sin \chi
\end{array}\right] U_{ \pm}^{( \pm)} \exp \left(-p A_{ \pm}\left|x_{3}\right|\right)} \\
Q_{ \pm}=C_{0}-\frac{1}{4}\left(\Omega_{+} \pm \Omega_{-}\right)^{2}, \quad Q_{+} Q_{-}=C_{0} m^{2} q^{2} \sin ^{2} \chi
\end{gathered}
$$

Function $\left(U_{ \pm}^{( \pm)}, U_{5}^{( \pm)}\right)$depends on $(p, q, \chi)$, and $( \pm)$ signifies $x_{3}>0(+), x_{3}<0(-)$. In view of (13) and (15) solution behavior is governed by the body wave speeds $\left(V_{4}, V_{5}=c_{5} V_{4}, V_{ \pm}=c_{ \pm} V_{4}\right)$, where $V_{-}<V_{+}$. Bounded behavior for $\hat{u}_{k}$ as $\left|x_{3}\right| \rightarrow \infty$ requires, in light of (16), that $\operatorname{Re}\left(A_{ \pm}\right)>0$ and $\operatorname{Re}\left(A_{5}\right)>0$ in the 
$q$-plane with, respectively, branch cuts

$$
\begin{aligned}
& \operatorname{Im}(q)=0, \quad \frac{-1}{c_{ \pm}-c}<\operatorname{Re}(q)<\frac{1}{c_{ \pm}+c}, \\
& \operatorname{Im}(q)=0, \quad \frac{-1}{c_{5}-c}<\operatorname{Re}(q)<\frac{1}{c_{5}+c} .
\end{aligned}
$$

It is noted that (17) is valid only so long as $c<\left(1, c_{5}\right)$, i.e., $V(\theta, \psi)<\left(V_{4}, V_{5}\right)$.

Expressions for $\left(U_{ \pm}^{( \pm)}, U_{5}^{( \pm)}\right)$in terms of $\left(\bar{\Delta}_{k}, \bar{\Sigma}_{k}\right)$ are obtained by combining (16) with the transforms . of (3b), (3c), and (6a). Details are found in Appendix B.

\section{Application to fracture problem: equations for solution}

Equation (16) represents the transform solution for the fracture problem if transforms of (5) are satisfied. Equation (5) does not involve $\Sigma_{k}$ so $\bar{\Sigma}_{k}$ can be dropped. Use of (7)-(12) and (B.3)-(B.5) and the transform of (3b), (3c), and (5) give three equations:

$$
\begin{gathered}
\bar{\sigma}_{31}^{C}=\frac{\mu p}{2 A_{5}}\left(S_{1} \bar{\Delta}_{1}+S_{12} \bar{\Delta}_{2}\right), \quad \bar{\sigma}_{32}^{C}=\frac{\mu p}{2 A_{5}}\left(s_{12} \bar{\Delta}_{1}+S_{2} \bar{\Delta}_{2}\right), \\
\bar{\sigma}_{33}^{C}-\frac{P}{p \beta}=\frac{\mu p}{2} A_{5} S_{3} \bar{\Delta}_{3} .
\end{gathered}
$$

Expressions for $\left(\bar{\sigma}_{31}^{C}, \bar{\sigma}_{32}^{C}\right)$ in (18a) can be combined to give, in addition,

$$
q^{2} \sin \psi \cos \psi \bar{\sigma}_{31}^{C}-Q_{5} \bar{\sigma}_{32}^{C}=\mu p\left(T_{1} \bar{\Delta}_{1}+T_{2} \bar{\Delta}_{2}\right) .
$$

Here, $\sigma_{3 k}^{C}$ is the traction generated ahead of crack $\left(\xi>0, c_{+} s-\xi-c s>0\right)$. Therefore, $\bar{\sigma}_{3 k}^{C}$ exists for $\operatorname{Re}(q)>-1 /\left(c_{+}-c\right)$. The second term in (18b) is transform of the Dirac function term in (5a), and therefore exists for $\operatorname{Re}(q)<1 / c$. Function $\Delta_{k}$ occurs for $\xi<0$ in a region generated behind wave front $c_{+} s+\xi+c s>0$, so that $\bar{\Delta}_{k}$ exists for $\operatorname{Re}(q)<1 /\left(c_{+}+c\right)$. Coefficients $Q_{5}$ and $\left(S_{1}, S_{12}, S_{2}, S_{3}, T_{1}, T_{2}\right)$ are defined in (B.1d) and Appendix C respectively.

\section{Wiener-Hopf equation}

The two equations in (18a) involve four unknowns $\left(\bar{\sigma}_{31}^{C}, \bar{\sigma}_{32}^{C}, \bar{\Delta}_{1}, \bar{\Delta}_{2}\right)$. In light of Appendix $C$ and remarks above, it is noted that pairs $\left(\bar{\sigma}_{31}^{C}, \bar{\sigma}_{32}^{C}\right)$ and $\left(\bar{\Delta}_{1}, \bar{\Delta}_{2}\right)$ have overlapping regions of analyticity in the complex $q$-plane, but coefficients $\left(S_{1}, S_{12}, S_{2}\right)$ do not. Nevertheless the two equations are homogeneous, which implies that

$$
\sigma_{3 k}^{C}=\Delta_{k}=0, \quad k=(1,2) .
$$

Nonhomogeneous (18b) involves only two unknowns $\left(\bar{\sigma}_{33}^{C}, \bar{\Delta}_{3}\right)$ with overlapping regions of analyticity. We therefore examine coefficients $\left(A_{5}, S_{3}\right)$ defined by (13) and (C.2) respectively. It is noted that

$$
\begin{gathered}
S_{3} \approx q^{2} R(c) \quad(|q| \rightarrow \infty), \\
S_{3}\left(q_{R}^{ \pm}\right)=0, \quad q_{R}^{ \pm}=\frac{ \pm 1}{c_{R} \pm c} .
\end{gathered}
$$


For a Category 1 material such that $c_{R}<c_{5}<c_{-}, R(c)$ is defined by

$$
\begin{gathered}
R(c)=\frac{1}{\sin ^{2} \chi-c^{2}}\left[4 d_{5}^{2} \cos ^{2} \chi+\frac{q_{5}^{2}}{\omega_{+} \omega_{-} a_{5}}\left(\frac{q_{-}}{a_{+}}-\frac{q_{+}}{a_{-}}\right)\right] \\
\quad+\frac{\sin ^{2} \chi}{\omega_{+} \omega_{-} a_{5}}\left[\frac{1}{a_{+}}\left(q_{+}-2 m q_{5}\right)-\frac{1}{a_{-}}\left(q_{+}-2 m q_{5}\right)\right], \\
a_{5}(c)=\frac{1}{\sqrt{d_{5}}} \sqrt{c_{5}^{2}-c^{2}}, \quad a_{ \pm}(c)=\sqrt{\cos ^{2} \chi-D_{\mp}^{2} / d_{1}}, \quad D_{ \pm}=\frac{1}{2}\left(\omega_{+} \pm \omega_{-}\right), \\
q_{5}(c)=d_{5} \cos ^{2} \chi+c_{5}^{2}-c^{2}, \quad q_{ \pm}(c)=\sin ^{2} \chi-c^{2}+D_{\mp}^{2} \\
\omega_{ \pm}(c)=\sqrt{\left(1+d_{1}\right) c^{2}-\Gamma \sin ^{2} \chi \pm 2 \sqrt{d_{1}\left(d_{2} \sin ^{2} \chi-c^{2}\right)\left(\sin ^{2} \chi-c^{2}\right)}}
\end{gathered}
$$

In (18b), $c_{R}=c_{R}(\theta, \psi)$ is the positive-real root of $R(c)$, where $0<c_{R}<c_{5},|\psi|<\pi / 2$, i.e., $V_{R}=$ $V_{R}(\theta, \psi)=c_{R}(\theta, \psi) V_{4}$ is the Rayleigh speed, and $\left(S_{3}, R\right)$ are Rayleigh functions for transverse isotropy. Subcritical crack extension therefore requires that $0<V<V^{*}=c^{*} V_{4}\left(0<c<c^{*}\right)$, where $c^{*}=$ $\min \left(1, c_{5}, c_{R}\right)$. It is also noted that $R \leq 0\left(0<c<c_{R}\right)$, and in view of (20) and (21), one can define function

$$
G_{3}=\frac{-S_{3}\left(c_{R}^{2}-c^{2}\right)}{R(c)\left[1+q\left(c_{R}-c\right)\right]\left[1-q\left(c_{R}+c\right)\right]}, \quad G_{3} \rightarrow 1(|q| \rightarrow \infty) .
$$

It has no roots and is analytic in the $q$-plane with branch cuts

$$
\begin{aligned}
& \operatorname{Im}(q)=0, \quad \frac{-1}{c_{5}-c}<\operatorname{Re}(q)<\frac{-1}{c_{+}-c} \\
& \operatorname{Im}(q)=0, \quad \frac{1}{c_{+}+c}<\operatorname{Re}(q)<\frac{1}{c_{5}+c}
\end{aligned}
$$

Function $G_{3}$ can therefore be written as product $G_{3}^{+} G_{3}^{-}$, where $G_{3}^{ \pm}$respectively are analytic in overlapping half-planes $\operatorname{Re}(q)>-1 /\left(c_{+}-c\right)$ and $\operatorname{Re}(q)<1 /\left(c_{+}+c\right)$. These functions are given in Appendix D. In (16b) one can write $A_{5}=A_{5}^{+} A_{5}^{-}$, where

$$
A_{5}^{+}=\frac{1}{d_{5}^{1 / 4}} \sqrt{1+q\left(c_{5}-c\right)}, \quad A_{5}^{-}=\frac{1}{d_{5}^{1 / 4}} \sqrt{1-q\left(c_{5}+c\right)} .
$$

Equation (23) indicates that $A_{5}^{ \pm}$respectively are analytic in overlapping half-planes $\operatorname{Re}(q)>-1 /\left(c_{5}-c\right)$ and $\operatorname{Re}(q)<1 /\left(c_{5}+c\right)$. In view of Appendix D, Equation (23) and the region of analyticity noted for $\left(\bar{\sigma}_{33}^{C}, \bar{\Delta}_{3}\right)$ and the second term in (18b), Equation (18b) itself can be put in the form of a Wiener-Hopf equation [Morse and Feshbach 1953]:

$$
\begin{aligned}
\frac{\bar{\sigma}_{33}^{C}}{\mu p} \frac{1}{A_{5}^{+} G_{3}^{+}} \frac{c_{R}-c}{1+q\left(c_{R}-c\right)}- & \frac{P}{\mu p^{2} \beta}\left[\frac{1}{A_{5}^{+} G_{3}^{+}} \frac{c_{R}-c}{1+q\left(c_{R}-c\right)}-\frac{d_{5}^{1 / 4} \sqrt{c}}{g_{3}^{+} \sqrt{c_{5}}}\left(1-\frac{c}{c_{R}}\right)\right] \\
& =-\frac{\bar{\Delta}_{3}}{2} \frac{R(c) A_{5}^{-} G_{3}^{-}}{c_{R}+c}\left[1-q\left(c_{R}+c\right)\right]+\frac{P}{\mu p^{2} \beta} \frac{d_{5}^{1 / 4} \sqrt{c}}{g_{3}^{+} \sqrt{c_{5}}}\left(1-\frac{c}{c_{R}}\right) \\
g_{3}^{+} & =G_{3}^{+}\left(\frac{1}{c}\right) .
\end{aligned}
$$


The left-hand and right-hand sides of (24a) are analytic in respectively the overlapping half-planes $\operatorname{Re}(q)>-1 /\left(c_{+}-c\right)$ and $\operatorname{Re}(q)<1 /\left(c_{+}+c\right)$ so that each side is an analytic continuation of the same entire function. In connection with (6) $\Delta_{3}$ must vanish continuously on $C$ for $x \rightarrow 0$. Equation (11a) therefore requires that $p q \bar{\Delta}_{k}$, and also the right-hand side of (24a), vanish for $|q| \rightarrow \infty$. The entire function itself must then in light of Liouville's theorem [Morse and Feshbach 1953] vanish, and (24a) leads to

$$
\begin{aligned}
& \bar{\Delta}_{3}=\frac{2 d_{5}^{1 / 4}}{\mu p^{2} \beta g_{3}^{+} R(c)} \sqrt{\frac{c}{c_{5}}}\left(1-\frac{c}{c_{R}}\right) \frac{1}{A_{5}^{-} G_{3}^{-}} \frac{c_{R}+c}{1-q\left(c_{R}+c\right)}, \\
& \bar{\sigma}_{33}^{C}=\frac{P}{p \beta}-\frac{P}{p \beta} \frac{d_{5}^{1 / 4}}{g_{3}^{+} c_{R}} \sqrt{\frac{c}{c_{5}}} G_{3}^{+} A_{5}^{+}\left[1+q\left(c_{R}-c\right)\right] .
\end{aligned}
$$

Examination of the fracture problem solution requires knowledge of $\left(\bar{\sigma}_{33}^{C}, \dot{\Delta}_{3}\right)$ for $x \rightarrow 0$ - and $x \rightarrow 0+$, respectively. In view of (7)-(9),

$$
\dot{f}=V_{4}\left(\partial-c \frac{\partial}{\partial \xi}\right) f
$$

Expressions for transforms $\left(\bar{\sigma}_{33}^{C}, p \beta \bar{\Delta}_{3}\right)$ that are valid for $|q| \rightarrow \infty$ suffice in this regard:

$$
\begin{aligned}
p \beta \bar{\Delta}_{3} & \approx \frac{2 P}{\mu p} \frac{\sqrt{d_{5} c}}{g_{3}^{+} R(c) \sqrt{c_{5}} \sqrt{c_{5}+c}}\left(1-\frac{c}{c_{R}}\right) \frac{1}{\sqrt{-q}}, \\
\bar{\sigma}_{33}^{C} & \approx \frac{P}{p} \frac{1}{g_{3}^{+} \sqrt{c}} \sqrt{1-\frac{c}{c_{5}}}\left(1-\frac{c}{c_{5}}\right) \frac{1}{\sqrt{q}} .
\end{aligned}
$$

\section{Transform inversions valid on crack plane near $C$}

For $1 / \sqrt{-q}$ and $1 / \sqrt{q}$, respectively, inverse operation (10b) yields

$$
\begin{gathered}
-\frac{p^{2}}{\pi \sqrt{-\xi}} \int_{+} \frac{d u}{\sqrt{u}} \exp (-p u) \quad(\xi<0), \\
-\frac{p^{2}}{\pi \sqrt{\xi}} \int_{+} \frac{d u}{\sqrt{u}} \exp (-p u) \quad(\xi>0) .
\end{gathered}
$$

The " + " signifies integration over the entire positive real $u$-axis. In view of (28), Equation (27) involves $p \exp (-p u)$, and its inverse is recognized as $\partial \delta(s-u)$ [Abramowitz and Stegun 1972]. The point force represents a step-function in time, so for generality we now consider the more general case

$$
P=P\left(V_{4} t\right), \quad P(0)=0 .
$$

Clarity of solution is enhanced if points in the crack plane are located with respect to fixed point $x_{0}$. Therefore the inverses of (27) are, by convolution, written as functions of $\left(\xi_{0}, \chi, s\right)$, where $\xi_{0}=\xi+c s$, and for $\left(s>0, \xi_{0} \rightarrow c s+,|\psi|<\pi / 2\right)$ :

$$
\dot{\Delta}_{3} \approx \frac{-2 V_{4}}{\mu \pi \sqrt{c s-\xi_{0}}} \frac{\sqrt{d_{5} c}}{g_{3}^{+} R(c) \sqrt{c_{5}} \sqrt{c_{5}+c}}\left(1-\frac{c}{c_{R}}\right) \partial \int_{0}^{s} \frac{d u}{\sqrt{s-u}} \frac{d P}{d u},
$$




$$
\sigma_{33}^{C} \approx \frac{1}{\pi \sqrt{c} \sqrt{\xi_{0}-c s}} \frac{1}{g_{3}^{+}} \sqrt{1-\frac{c}{c_{5}}}\left(1-\frac{c}{c_{R}}\right) \partial \int_{0}^{s} \frac{d u}{\sqrt{s-u}} \frac{d P}{d u} .
$$

\section{Transform inversions valid near $C$}

Expressions for $\dot{u}_{k}$ near $C$ for $\left(x_{3}^{0}, x_{3}\right) \neq 0$ are also required. In view of (7c) and (26), $L\left(\dot{u}_{k}\right)=\beta p \bar{u}_{k}$ and $\bar{u}_{k}$ can be generated from (15) and Appendix B by setting $\left(\bar{\Sigma}_{k}, \bar{\Delta}_{1}, \bar{\Delta}_{2}\right)=0$ and using (25a). For $x_{3} \neq 0$ a more explicit version of inversion formula (10) is useful:

$$
\begin{aligned}
\tilde{f}\left(p, q_{1}, q_{2}, x_{3}\right) & \rightarrow f_{\Psi}(p, q, \chi) \exp \left(-p \mathrm{~A}\left|x_{3}\right|\right), \quad \mathrm{A}=\left(A_{ \pm}, A_{5}\right), \\
\hat{f}\left(p, \xi, \chi, x_{3}\right) & =-\frac{p^{2}}{2 \pi} \int \frac{|q|}{q} f_{\Psi}(p, q, \chi) \exp \left[p\left(q \xi-\mathrm{A}\left|x_{3}\right|\right)\right] d q .
\end{aligned}
$$

Result (28) follows from use of Cauchy theory to change the integration path in (10b) to the $\operatorname{Re}(q)$-axis. For (31b) the path is changed to a contour $q(\mathrm{~A})$ in the complex $q$-plane along which the exponential term assumes the form $\exp (-p u)$, where $u$ is real and positive. Because inversions valid near $C$ are sufficient, local coordinates $(r, \psi, \phi)$, centered on the portion of $C$ that borders $\delta A$, are introduced, where

$$
r=\sqrt{\xi^{2}+x_{3}^{2}}, \quad \phi=\tan ^{-1} \frac{x_{3}}{\xi} \quad(|\phi|<\pi) .
$$

In (31b) $q\left(A_{ \pm}\right)$and $q\left(A_{5}\right)$ for $r \approx 0$ have, respectively, asymptotic forms

$$
\begin{array}{ll}
-\frac{u}{r S_{ \pm}}, & S_{ \pm}=\cos \phi \mp i a_{ \pm} \sin \phi, \\
-\frac{u}{r S_{5}}, & S_{5}=\cos \phi \mp i a_{5} \sin \phi .
\end{array}
$$

It is noted that (25a), which depends on $1 / \sqrt{-q}$, is associated in Appendix B with operator $( \pm)$. In the case of contour $q\left(A_{5}\right)$ therefore, (28a) and (28b), respectively, are replaced by

$$
\text { (干) } \frac{p}{\pi} \frac{\mathrm{A}_{5}^{(-)}}{\sqrt{2 r}} \int_{+} \frac{d u}{\sqrt{u}} \exp (-p u), \quad-\frac{p}{\pi} \frac{\mathrm{A}_{5}^{(+)}}{\sqrt{2 r}} \int_{+} \frac{d u}{\sqrt{u}} \exp (-p u) .
$$

In the case of contour $q\left(A_{ \pm}\right)$, replacements are

$$
\text { (干) } \frac{p}{\pi} \frac{\mathrm{A}_{ \pm}^{(-)}}{\sqrt{2 r}} \int_{+} \frac{d u}{\sqrt{u}} \exp (-p u), \quad-\frac{p}{\pi} \frac{\mathrm{A}_{ \pm}^{(+)}}{\sqrt{2 r}} \int_{+} \frac{d u}{\sqrt{u}} \exp (-p u) .
$$

In (34), $\left(A_{5}^{( \pm)}, A_{ \pm}^{( \pm)}\right)$are factors of the real $(+)$and imaginary (-) parts of $\left(\sqrt{S_{5}}, \sqrt{S_{ \pm}}\right)$:

$$
\begin{array}{lll}
A_{5}^{( \pm)}=\sqrt{1( \pm) \cos \phi / B_{5}}, & B_{5}=\sqrt{1-m_{5} \sin ^{2} \phi}, \\
A_{ \pm}^{( \pm)}=\sqrt{1( \pm) \cos \phi / B_{ \pm}}, & B_{ \pm}=\sqrt{1-m_{ \pm} \sin ^{2} \phi}, \\
m_{5}=\sin ^{2} \chi-\frac{1}{d_{5}}\left(\sin ^{2} \chi-c^{2}\right), & m_{ \pm}=\sin ^{2} \chi+\frac{D_{\mp}^{2}}{d_{1}} .
\end{array}
$$


Use of (32)-(35) gives for $(r \rightarrow 0,|\psi|<\pi / 2,|\phi|<\pi)$

$$
\dot{u}_{1} \approx-\frac{K_{3} \mathrm{M}_{1}}{\mu \sqrt{2 r}} \cos \chi, \quad \dot{u}_{2} \approx \frac{K_{3}}{\mu \sqrt{2 r}} \frac{\mathrm{M}_{2}}{\sin \chi}, \quad \dot{u}_{3} \approx( \pm) \frac{K_{3} \mathrm{M}_{3}}{\mu \sqrt{2 r}} .
$$

In (36) coefficient $\mathrm{M}_{k}$ is

$$
\begin{aligned}
& \mathrm{M}_{1}=\frac{d_{5} a_{5} \mathrm{~A}_{5}^{(+)}}{\sin ^{2} \chi-c^{2}}+\frac{1}{2 \omega_{+} \omega_{-}}\left(\mathrm{N}_{-} \frac{\mathrm{A}_{+}^{(+)}}{a_{+}}-\mathrm{N}_{+} \frac{\mathrm{A}_{-}^{(+)}}{a_{-}}\right), \\
& \mathrm{M}_{2}=\frac{1}{2 m \omega_{+} \omega_{-}}\left(\frac{q_{+} \mathrm{N}_{-}}{\sin \chi} \frac{\mathrm{A}_{+}^{(+)}}{a_{+}}-\frac{q_{-} \mathrm{N}_{+}}{\sin \chi} \frac{\mathrm{A}_{-}^{(+)}}{a_{-}}\right), \\
& \mathrm{M}_{3}=\frac{d_{5} \mathrm{~A}_{5}^{(-)} \cos ^{2} \chi}{\sin ^{2} \chi-c^{2}}+\frac{1}{2 \omega_{+} \omega_{-}}\left(\mathrm{N}_{-} \mathrm{A}_{+}^{(-)}-\mathrm{N}_{+} \mathrm{A}_{-}^{(-)}\right) .
\end{aligned}
$$

In (37) $\left(K_{3}, \mathrm{~N}_{ \pm}\right)$are defined by

$$
\begin{aligned}
K_{3} & =\frac{2 V_{4}}{\pi g_{3}^{+} R(c) \sqrt{c_{5}+c}} \sqrt{d_{5} / c_{5}}\left(1-\frac{c}{c_{R}}\right) \partial \int_{0}^{s} \frac{d u}{\sqrt{s-u}} \frac{d P}{d u} \\
\mathrm{~N}_{ \pm} & =m \sin ^{2} \chi-\frac{q_{5} q_{ \pm}}{\sin ^{2} \chi-c^{2}}
\end{aligned}
$$

\section{Criterion: dynamic energy release rate}

A standard criterion for brittle fracture (see, e.g., [Freund 1972]) equates the rate at which surface energy is released to the rate of work associated with traction and relative displacements in the fracture zone $F$. If kinetic energy is included [Gdoutos 2005] the equation for this problem takes the form

$$
D \iint_{\partial A} e_{F} d x_{1}^{0} d x_{2}^{0}=\iint_{F} \sigma_{33}^{C} \dot{\Delta}_{3} d x_{1}^{0} d x_{2}^{0}+D \iiint_{123} \frac{1}{2} \rho \dot{u}_{k} \dot{u}_{k} d x_{1}^{0} d x_{2}^{0} d x_{3}^{0} .
$$

Here $e_{F}$ is the surface energy per unit area, and is generally assumed to be constant [de Boer et al. 1988; Skriver and Rosengaard 1992]. Fracture zone $F$ is a strip of infinitesimal thickness in the $x_{1}^{0} x_{2}^{0}$-plane that straddles the portion of $C$ that borders $\delta A$. Subscript 123 signifies integration over the solid. Use of transport theory [Malvern 1969] and translating basis $x$ expressed in terms of $\left(\xi, \psi, x_{3}=0\right)$ gives for the first term in (39)

$$
V_{4} e_{F} s \int_{\Psi} c \sqrt{c^{2}+\left(c^{\prime}\right)^{2}}, \quad f^{\prime}=\frac{d f}{d \psi} .
$$

Here $\Psi$ signifies integration over $|\psi|<\pi / 2$. Use of $x$ for the integration over $F$ gives

$$
\int_{\Psi} d \Psi \int_{c s-}^{c s+}\left|x_{0}\right| \sigma_{33}^{C} \dot{\Delta}_{3} d x_{0}
$$

In light of (30) it can be shown [Freund 1972] that the integrand of (41a) features Dirac function $\delta\left(\xi_{0}-c s\right)$. Moreover, linear behavior in $s$ displayed in (40) places a restriction on $\partial_{s} P$; see, e.g., [Achenbach and Brock 1973]. That is, $V$ must in general vary with time. One case, however, for which time-invariance is valid is

$$
\partial P=p_{C} \sqrt{s} \quad\left(p_{C}>0\right) .
$$


Equation (30) and (41a) then give

$$
-\pi p_{C}^{2} \frac{s}{\pi} \int_{\Psi} V d \psi \sqrt{\frac{c_{5}-c}{c_{5}+c}} K_{C}^{2} R(c), \quad K_{C}=\frac{-\sqrt{d_{5}}}{c_{5} g_{3}^{+} R(c)}\left(1-\frac{c}{c_{R}}\right) .
$$

Equation (36) is singular near $C$. The last integration in (39) can then be, via transport theory [Malvern. 1969], taken over the surface of a tube of radius $r_{C} \rightarrow 0$ that encloses the portion of $C$ that borders $\delta A$. Integration can be performed in terms of coordinates (29) and expressions (32), (37), (38), and (41b). The last term in (39) becomes

$$
\begin{gathered}
-s \frac{P_{C}^{2}}{\mu} \int_{\Psi} V d \psi \sqrt{c^{2}+\left(c^{\prime}\right)^{2}} \frac{c_{5} K_{C}^{2}}{c_{5}+c} \int_{\Phi} \mathrm{E}_{\Phi} \cos \phi d \phi, \\
\mathrm{E}_{\Phi}=\mathrm{M}_{1}^{2} \cos ^{2} \chi+\frac{\mathrm{M}_{2}^{2}}{\sin ^{2} \chi}+\mathrm{M}_{3}^{2} .
\end{gathered}
$$

Here $\Phi$ signifies integration over range $|\phi|<\pi$. Equation (40), (41c), and (42) all involve integration with respect to $\psi$, so that (39) gives for $|\psi|<\pi / 2$

$$
\sqrt{c^{2}+\left(c^{\prime}\right)^{2}}+\pi \frac{p_{C}^{2} K_{C}^{2}}{\mu e_{F}} \sqrt{\frac{c_{5}-c}{c_{5}+c}} R(c)-\frac{p_{C}^{2} K_{C}^{2}}{4 \mu e_{F} c_{5}} \frac{d_{5} c}{c_{5}+c} \sqrt{c^{2}+\left(c^{\prime}\right)^{2}} \int_{\Phi} \mathrm{E}_{\Phi} \cos \phi d \phi=0
$$

\section{Differential equation: observations}

Here (43) is a nonlinear differential equation for $c$. As viewed in coordinates aligned with the initial rectilinear crack edge $c=c(\theta, \psi)=c(\chi)$. Equation (37) and (42b) show that the integrand of (43) involves quadratics in $\left(A_{ \pm}^{(+)}, A_{5}^{(+)}\right)$and quadratics in $\left(A_{ \pm}^{(-)}, A_{5}^{(-)}\right)$. Use of (35) and (37) shows that

$$
\begin{aligned}
& \int_{\Phi}\left[\left(\mathrm{A}_{ \pm}^{(+)}\right)^{2}+\left(\mathrm{A}_{ \pm}^{(-)}\right)^{2}\right] \cos \phi d \phi=0, \quad \int_{\Phi}\left[\left(\mathrm{A}_{5}^{(+)}\right)^{2}+\left(A_{5}^{(-)}\right)^{2}\right] \cos \phi d \phi=0, \\
& \int_{\Phi}\left[\mathrm{A}_{+}^{(+)} \mathrm{A}_{-}^{(+)}+\mathrm{A}_{+}^{(-)} \mathrm{A}_{-}^{(-)}\right] \cos \phi d \phi=0, \quad \int_{\Phi^{\prime}}\left[\mathrm{A}_{ \pm}^{(+)} \mathrm{A}_{5}^{(+)}+\mathrm{A}_{ \pm}^{(-)} \mathrm{A}_{5}^{(-)}\right] \cos \phi d \phi=0 .
\end{aligned}
$$

Use of (42b) and (44) in (43) gives

$$
\begin{gathered}
\int_{\Phi} \mathrm{E}_{\Phi} \cos \phi d \phi=\frac{\pi}{4} \mathrm{E}(c) \\
\mathrm{E}(c)=\mathrm{T}_{+}\left(\frac{\mathrm{N}_{-}}{a_{+} \omega_{+} \omega_{-}}\right)^{2}\left[\frac{1}{m^{2} \sin ^{2} \chi}\left(\sin ^{2} \chi-c^{2}+D_{-}^{2}\right)^{2}+\frac{D_{-}^{2}}{d_{1}}\right] \\
+\mathrm{T}_{-}\left(\frac{\mathrm{N}_{+}}{a_{-} \omega_{+} \omega_{-}}\right)^{2}\left[\frac{1}{m^{2} \sin ^{2} \chi}\left(\sin ^{2} \chi-c^{2}+D_{+}^{2}\right)^{2}+\frac{D_{+}^{2}}{d_{1}}\right] \\
-2 \mathrm{~T}_{+}^{-} \frac{\mathrm{N}_{+} \mathrm{N}_{-}}{\omega_{+}^{2} \omega_{-}^{2}}\left[\frac{\sqrt{d_{1}}\left(1-c^{2}\right)}{\sqrt{c_{+}^{2}-c^{2}} \sqrt{c_{-}^{2}-c^{2}}}-1\right]+4 \mathrm{~T}_{5} \frac{d_{5} \cos ^{4} \chi}{\sin ^{2} \chi-c^{2}} \\
+\frac{2 d_{5} \cos ^{2} \chi}{\omega_{+} \omega_{-}\left(\sin ^{2} \chi-c^{2}\right)}\left[\mathrm{T}_{5}^{+} \mathrm{N}_{-}\left(\frac{a_{5}}{a_{+}}-1\right)-\mathrm{T}_{5}^{-} \mathrm{N}_{+}\left(\frac{a_{5}}{a_{-}}-1\right)\right] .
\end{gathered}
$$


Equation (45b) involves terms

$$
\begin{aligned}
& \mathrm{T}_{ \pm}=\frac{1}{\pi} \int_{\Phi} d \phi \frac{\cos ^{2} \phi}{\sqrt{1-m_{ \pm} \sin ^{2} \phi}}, \quad \mathrm{T}_{5}=\frac{1}{\pi} \int_{\Phi} d \phi \frac{\cos ^{2} \phi}{\sqrt{1-m_{5} \sin ^{2} \phi}} \\
& \mathrm{T}_{+}^{-}=\frac{1}{\pi} \int_{\Phi} d \phi \cos \phi \sqrt{1+\frac{\cos \phi}{\sqrt{1-m_{+} \sin ^{2} \phi}}} \sqrt{1+\frac{\cos \phi}{\sqrt{1-m_{-} \sin ^{2} \phi}}} \\
& \mathrm{T}_{5}^{ \pm}=\frac{1}{\pi} \int_{\Phi} d \phi \cos \phi \sqrt{1+\frac{\cos \phi}{\sqrt{1-m_{ \pm} \sin ^{2} \phi}}} \sqrt{1+\frac{\cos \phi}{\sqrt{1-m_{5} \sin ^{2} \phi}}}
\end{aligned}
$$

Equation (46a) gives complete elliptic integrals of the first $(F)$ and second $(E)$ kind when $0<\left(m_{ \pm}, m_{5}\right)<1$, for example,

$$
\mathrm{T}_{5}=4\left[F\left(\sqrt{m_{5}}\right)+\frac{1}{m_{5}}\left(E\left(\sqrt{m_{5}}\right)-F\left(\sqrt{m_{5}}\right)\right)\right] .
$$

Equation (43) is somewhat complicated and opaque. Insight concerning $c(\theta, \psi)$, based partly on analytical expressions, is possible by considering values of parameter $V(\theta, \psi)$ that are not negligible, but well below critical, i.e., $c / c^{*} \leq 0.3$. Thus (43) is replaced with

$$
\sqrt{c^{2}+\left(c^{\prime}\right)^{2}}\left(1+\frac{\pi p_{C}^{2}}{4 \mu e_{F}} \frac{d_{5}}{c_{5}} \frac{c \mathrm{E}(0)}{4 R^{2}(0)}\right)+\frac{\pi p_{C}^{2}}{\mu e_{F} R(0)} \frac{\sqrt{d_{5}}}{c_{5}} \approx 0 .
$$

Parameter $c$ in $(R, \mathrm{E})$ has been dropped and, in particular,

$$
\begin{gathered}
\mathrm{N}_{ \pm}=m \sin ^{2} \chi-\left(1-d_{\mp}^{2}\right)\left(c_{5}^{2}+d_{5} \cos ^{2} \chi\right), \\
a_{ \pm}=\sqrt{\cos ^{2} \chi+d_{\mp}^{2} / d_{1} \sin ^{2} \chi}, \quad a_{5}=\frac{c_{5}}{\sqrt{d_{5}}}, \quad q_{5}=c_{5}^{2}+d_{5} \cos ^{2} \chi, \\
d_{ \pm}=\frac{1}{2}\left(\sqrt{\Gamma+2 \sqrt{d_{1} d_{2}}} \pm \sqrt{\Gamma-2 \sqrt{d_{1} d_{2}}}\right), \quad d=\sqrt{\Gamma^{2}-4 d_{1} d_{2}}, \\
m_{ \pm}=\left(1-d_{\mp}^{2} / d_{1}\right) \sin ^{2} \chi, \quad m_{5}=\left(1-1 / d_{5}\right) \sin ^{2} \chi .
\end{gathered}
$$

Equation (48c) indicates that attention henceforth focuses on Category 1 materials. However, the additional restriction that was placed on (21a) can be dropped.

\section{Study of differential equation approximation}

Equation (47) is also a nonlinear differential equation, but explicit in $\left(c, c^{\prime}\right)$. Terms $R(0), \mathrm{E}(0)$, and (48) are explicit functions of $\left(\cos ^{2} \chi, \sin ^{2} \chi\right)$, so that an analytic solution may be difficult, but after [Brock $2017 \mathrm{a} ; 2017 \mathrm{~b}]$ an approximate solution is feasible:

$$
c(\theta, \psi)=c(\chi) \approx \sum_{0}^{N} b_{k} \cos ^{2 k} \chi \quad(0<|\psi|<\pi / 2) .
$$

Only the case $\theta=0$ is treated in [Brock 2017a; 2017b] and expressions for coefficients which correspond to $b_{k}$ are obtained by direct substitution into the nonlinear differential equation, with coefficients expanded in powers of $\cos ^{2} \psi$. The first three terms $\left(b_{0}, b_{1}, b_{2}\right)$ are found to give results that are generally accurate to within three significant figures. Here dependence of $R(0), \mathrm{E}(0)$, and (48) on parameter 
$\chi=\theta+\psi$ is more complicated. Therefore $N+1$ equations for $b_{k}$ are obtained by substitution of (49) into (47) for $N+1$ values of $\chi$. We here choose for illustration $N=2,0<\theta<\pi / 2$ and values $\chi(0, \pi / 2, \pi / 4)$. The corresponding values for $(R(0), \mathrm{E}(0))$ are obtained from (21a) and (45b) as $\left(R_{0}, \mathrm{E}_{0}\right),\left(R_{2}, \mathrm{E}_{2}\right)$, and $\left(R_{4}, \mathrm{E}_{4}\right)$, respectively, and are found in Appendix $\mathrm{E}$. If kinetic energy is neglected by dropping the $\mathrm{E}-$ terms the three simultaneous equations give

$$
\begin{gathered}
b_{0}=q_{2} \\
b_{1}=-3 q_{2}-q_{0}+2 \sqrt{q_{4}^{2}-\left(q_{0}-q_{2}\right)^{2}}, \\
b_{2}=2\left[q_{0}+q_{2}-2 \sqrt{q_{4}^{2}-\left(q_{0}-q_{2}\right)^{2}}\right], \\
q_{0}=\frac{\pi p_{C}^{2}}{\mu e_{F}\left|R_{0}\right|}, \quad q_{2}=\frac{\pi p_{C}^{2}}{\mu e_{F}} \frac{\sqrt{d_{5}}}{\left|R_{2}\right|}, \quad q_{4}=\frac{\pi p_{C}^{2}}{\mu e_{F}} \frac{\sqrt{2 d_{5}}}{\left|R_{4}\right| \sqrt{1+d_{5}}} .
\end{gathered}
$$

Inclusion of kinetic energy gives for $b_{0}$

$$
b_{0}=\frac{1}{2 Q_{2}}\left(1-\sqrt{1-4 q_{2} Q_{2}}\right)
$$

Expressions for $\left(b_{1}, b_{2}\right)$ are obtained in light of (51a) from the simultaneous solution of a linear and a fourth-order algebraic equation:

$$
\begin{gathered}
b_{1}+b_{2}=q_{12}=\frac{1}{2 Q_{0}}\left(1-\sqrt{1-4 q_{0} Q_{0}}\right)-b_{0} \\
{\left[\left(q_{12}^{0}\right)^{2}+q_{12}^{2}\right]\left(\frac{1}{Q_{4}}-q_{12}^{0}\right)^{2}=\left(\frac{q_{4}}{Q_{4}}\right)^{2}, \quad q_{12}^{0}=b_{0}+\frac{1}{4}\left(b_{1}+q_{12}\right) .}
\end{gathered}
$$

In (51a)-(51c), Equation (50d) holds and

$$
Q_{0}=\frac{\pi p_{C}^{2}}{\mu e_{F}} \frac{\sqrt{d_{5}}\left|\mathrm{E}_{0}\right|}{4 R_{0}^{2}}, \quad Q_{2}=\frac{\pi p_{C}^{2}}{\mu e_{F}} \frac{d_{5}\left|\mathrm{E}_{2}\right|}{4 R_{2}^{2}}, \quad Q_{4}=\frac{\pi p_{C}^{2}}{\mu e_{F}} \frac{\sqrt{2} d_{5}}{\sqrt{1+d_{5}}} \frac{\left|\mathrm{E}_{4}\right|}{4 R_{4}^{2}} .
$$

\section{Sample calculations: wave speeds}

Consider a Category 1 material, similar to beryl, with properties [Payton 1983; de Boer et al. 1988; Skriver and Rosengaard 1992]:

$$
\begin{gathered}
C_{44}=\mu=6.894 \mathrm{GPa}, \quad e_{F}=2.0 \mathrm{~J} / \mathrm{m}^{2}, \quad V_{4}=1569 \mathrm{~m} / \mathrm{s}, \\
d_{1}=4.11, \quad d_{2}=3.62, \quad d_{5}=2.0, \quad m=2.017, \quad \Gamma=11.81
\end{gathered}
$$

Equation (13) and (15a), respectively, define dimensionless body wave speeds $\left(c_{5}, c_{ \pm}\right)$, and dimensionless Rayleigh speed $c_{R}$ is a root of $R(c)$, defined in (21). They, and dimensionless crack speed parameter $c$, arise in the solution as viewed from the frame aligned with the original rectilinear crack edge. That is, they are functions of $\chi$. For the Category 1 material chosen here, values of these dimensionless speeds are listed in Table 1 for various $\chi$, (given for clarity in degrees ${ }^{\circ}$ ). Experience, e.g., [Brock 2013], indicates that factorizations of $R(c)$ may or may not arise for transverse isotropy. Thus the actual root-exhibiting 
functions for given $\psi$ are distinct in form. When $\chi=0$ and $\chi=\pi / 2$ respectively, for example,

$$
\begin{aligned}
4 d_{5} \sqrt{d_{5}-c^{2}} \sqrt{d_{1}-c^{2}}-\sqrt{d_{1} / d_{5}}\left(2 d_{5}-c^{2}\right)^{2}=0, & c=c_{R}=1.245, \\
{\left[d_{1}\left(d_{2}-c^{2}\right)-(m-1)^{2}\right] \sqrt{1-c^{2}}-\sqrt{d_{1}} c^{2} \sqrt{d_{2}-c^{2}}=0, } & c=c_{R}=0.954 .
\end{aligned}
$$

In addition to anisotropic behavior exhibited in Table 1 , location of speed parameter $c^{*}$ shows that critical speed is not necessarily the Rayleigh wave value. In contrast to isotropic materials [Achenbach 1973], transonic crack extension rates may define the onset of critical behavior. This feature is noted in 3D analyses of other problems in transversely isotropic materials, e.g., sliding contact in the dynamic steady state [Brock 2013].

\section{Sample calculations: crack extension rate parameters}

For illustration in terms of the Category 1 material properties listed above, we consider a loading parameter $p_{C}=3\left(10^{4}\right) \mathrm{N} / \mathrm{m}^{3 / 2}$ that is consistent with small values of speed parameter $V$. For this value and the Category 1 material featured in Table 1, (49) and (50) give the approximation valid when kinetic energy is neglected:

$$
c \approx 0.12454-0.03404 \cos ^{2}(\theta+\psi)+0.00937 \cos ^{4}(\theta+\psi) .
$$

Here $(0<\theta<\pi / 2, \psi<\pi / 2)$. For the kinetic energy case, (49) and (51) give

$$
c \approx 0.1249-0.0217 \cos ^{2}(\theta+\psi)-0.0032 \cos ^{4}(\theta+\psi) .
$$

In (52), $0<\theta<\pi / 2, \psi<\pi / 2$ and it is noted that successive term coefficients decrease by orders of magnitude. Calculations based on (52a) and (52b) are displayed in Table 2 and Table 3, respectively.

As in Table 1 anisotropic behavior is prominent. In particular the newly created crack surface $\delta A$ is a semioval that is symmetric only when the original rectilinear crack edge coincides with the (principal) $x_{2}^{0}$-direction. In the isotropic limit [Brock 2017b] $\delta A$ is semicircular. Table 2 and Table 3 data also show that neglect of kinetic energy leads to an under-prediction of crack extension rate. The effect is however not large. Indeed, a more pronounced kinetic energy effect arises for crack extension in an isotropic, thermoelastic material [Brock 2017a].

\begin{tabular}{ccccc}
$\chi=\theta+\psi$ & $c_{5}$ & $c_{+}$ & $c_{-}$ & $c_{R}$ \\
\hline $0^{\circ}$ & $\sqrt{2}$ & 2.027 & $1.0^{*}$ & 1.245 \\
$15^{\circ}$ & 1.39 & 1.998 & 1.042 & 1.226 \\
$30^{\circ}$ & 1.323 & 1.925 & 1.133 & 1.172 \\
$45^{\circ}$ & 1.225 & 1.857 & 1.19 & 1.099 \\
$60^{\circ}$ & 1.118 & 1.851 & 1.147 & 1.027 \\
$75^{\circ}$ & 1.034 & 1.886 & 1.048 & $0.974^{*}$ \\
$90^{\circ}$ & 1.0 & 1.903 & 1.0 & $0.954^{*}$
\end{tabular}

Table 1. Body $\left(c_{5}, c_{ \pm}\right)$and Rayleigh $\left(c_{R}\right)$ wave speed parameters $\left({ }^{*}\right.$ signifies critical value $c^{*}$ ). 


\begin{tabular}{rcccc}
$\psi \psi$ & $c:\left(\theta=0^{\circ}\right)$ & $c:\left(\theta=30^{\circ}\right)$ & $c:\left(\theta=45^{\circ}\right)$ & $c:\left(\theta=60^{\circ}\right)$ \\
\hline$-90^{\circ}$ & 0.1245 & 0.1166 & 0.1099 & 0.1043 \\
$-75^{\circ}$ & 0.1223 & 0.1099 & 0.1043 & 0.1009 \\
$-60^{\circ}$ & 0.1166 & 0.1043 & 0.1009 & 0.0999 \\
$-45^{\circ}$ & 0.1099 & 0.1009 & 0.0999 & 0.1009 \\
$-30^{\circ}$ & 0.1043 & 0.0999 & 0.1009 & 0.1043 \\
$-15^{\circ}$ & 0.1009 & 0.1009 & 0.1043 & 0.1099 \\
$0^{\circ}$ & 0.0999 & 0.1043 & 0.1099 & 0.1166 \\
$15^{\circ}$ & 0.1009 & 0.1099 & 0.1166 & 0.1223 \\
$30^{\circ}$ & 0.1043 & 0.1166 & 0.1223 & 0.1245 \\
$45^{\circ}$ & 0.1099 & 0.1223 & 0.1245 & 0.1223 \\
$60^{\circ}$ & 0.1166 & 0.1245 & 0.1223 & 0.1166 \\
$75^{\circ}$ & 0.1223 & 0.1223 & 0.1166 & 0.1099 \\
$90^{\circ}$ & 0.1245 & 0.1166 & 0.1099 & 0.1043
\end{tabular}

Table 2. Dimensionless speed $c$ for $p_{C}=3\left(10^{4}\right) \mathrm{N} / \mathrm{m}^{3 / 2}$ (kinetic energy neglected).

\begin{tabular}{rcccc}
$\psi$ & $c:\left(\theta=0^{\circ}\right)$ & $c:\left(\theta=30^{\circ}\right)$ & $c:\left(\theta=45^{\circ}\right)$ & $c:\left(\theta=60^{\circ}\right)$ \\
\hline$-90^{\circ}$ & 0.1249 & 0.1193 & 0.1132 & 0.1068 \\
$-75^{\circ}$ & 0.1233 & 0.1132 & 0.1068 & 0.1018 \\
$-60^{\circ}$ & 0.1193 & 0.1068 & 0.1018 & 0.0999 \\
$-45^{\circ}$ & 0.1132 & 0.1018 & 0.0999 & 0.1018 \\
$-30^{\circ}$ & 0.1068 & 0.0999 & 0.1018 & 0.1068 \\
$-15^{\circ}$ & 0.1018 & 0.1018 & 0.1068 & 0.1132 \\
$0^{\circ}$ & 0.0999 & 0.1068 & 0.1132 & 0.1193 \\
$15^{\circ}$ & 0.1018 & 0.1132 & 0.1193 & 0.1233 \\
$30^{\circ}$ & 0.1068 & 0.1193 & 0.1233 & 0.1249 \\
$45^{\circ}$ & 0.1132 & 0.1233 & 0.1249 & 0.1233 \\
$60^{\circ}$ & 0.1193 & 0.1249 & 0.1233 & 0.1193 \\
$75^{\circ}$ & 0.1233 & 0.1233 & 0.1193 & 0.1132 \\
$90^{\circ}$ & 0.12488 & 0.1193 & 0.1132 & 0.1068
\end{tabular}

Table 3. Dimensionless speed $c$ for $p_{C}=3\left(10^{4}\right) \mathrm{N} / \mathrm{m}^{3 / 2}$ (kinetic energy included).

\section{Some observations}

This paper complements [Brock 2017a; 2017b] by considering 3D transient fracture at the edge of an initially undisturbed, closed semi-infinite slit in a transversely isotropic solid. Loading, however, is by point-force compression at the slit edges, whereas mixed-mode point-force loading at the edges and isotropic solids are treated in [Brock 2017a; 2017b]. Pure compression generates a semicircular crack edge in the isotropic solids, and shear loading distorts the profile. One goal of this paper is to illustrate the effects of anisotropy itself on crack profile. To this end, therefore, the case of the material rotational 
symmetry axis is parallel to the crack plane, and the initially rectilinear crack edge does not align with a principal axis in that plane.

As in [Brock 2017a; 2017b] kinetic energy is included in imposition of the dynamic energy release rate criterion, and effects of inclusion on solution response is examined. Moreover, the same type of point-force time dependence is treated that guarantees dynamic similarity, i.e., the crack edge profile might not be circular, but is not time-dependent. The effect of anisotropy is seen to indeed be clear: pure compression loading creates a semioval crack profile. The under-prediction of crack edge extension rates caused by neglect of kinetic energy is also seen, although it is not as pronounced as that noted in [Brock 2017a; 2017b].

The rapid growth in time of the dynamic similarity-inducing point force may not be realistic. For example the selection for $p_{C}$ and the Category 1 material used here produce in light of (41b) point-force function

$$
P=1.243\left(10^{9}\right)(t / \mathrm{s})^{3 / 2} \mathrm{~N} .
$$

Here $t$ is time after initiation given in seconds (s), indicating that $P$ rapidly achieves large values. Nevertheless, if $P$ represents the short-time behavior of the point forces, the present analysis and those in [Brock 2017a; 2017a] can be valid for the study of transient fracture initiation. The short-time limitation is in fact imposed during the development of results in [Brock 2017a].

This paper is not a definitive study of the canonical problem of the semi-infinite slit in an unbounded, transversely isotropic material with point-force loading. In particular, absence of shear loading restricted the fracture process to the crack-opening mode. Moreover, some key expressions and associated calculations are based on a particular type of transversely isotropic material [Payton 1983]. Nevertheless, the paper is offered as a starting point and check for more ambitious efforts.

\section{Appendix A}

$$
\text { Category 1: } \begin{aligned}
2 \sqrt{d_{1} d_{2}} & \leq \Gamma \leq 1+d_{1} d_{2} & & \left(1<d_{1}<d_{2}\right), \\
d_{1}+d_{2} & \leq \Gamma \leq 1+d_{1} d_{2} & & \left(1<d_{2}<d_{1}\right), \\
2 d_{1} & \leq \Gamma \leq 1+d_{1}^{2} & & \left(1<d_{2}=d_{1}\right),
\end{aligned}
$$

Category 2: $\quad 1+d_{1}<\Gamma<d_{1}+d_{2} \quad\left(\Gamma^{2}-4 d_{1} d_{2}<0\right)$,

Category 3: $\quad \Gamma<1+d_{1} \quad\left(\Gamma^{2}-4 d_{1} d_{2}<0\right)$.

\section{Appendix B}

Transform of (6a) gives six equations that uncouple into two sets. In Set 1,

$$
\begin{gathered}
\mathrm{P}_{5}-m q^{2} \sin \chi \cos \chi\left(\mathrm{P}_{+}+\mathrm{P}_{-}\right)=\bar{\Delta}_{1}, \\
Q_{+} \mathrm{P}_{+}+Q_{-} \mathrm{P}_{-}=\bar{\Delta}_{2}, \\
-2 d_{5} q \cos \chi \mathrm{P}_{5}+q \sin \chi\left[\left(m Q_{5}-Q_{+}\right) \mathrm{P}_{+}+\left(m Q_{5}-Q_{-}\right) \mathrm{P}_{-}\right]=\bar{\Sigma}_{3} /(\mu p), \\
\mathrm{P}_{5}=A_{5}\left(U_{5}^{(+)}+U_{5}^{(-)}\right), \quad \mathrm{P}_{ \pm}=U_{ \pm}^{(+)}+U_{ \pm}^{(-)}, \quad Q_{5}=C_{0}+2 d_{5} q^{2} \cos ^{2} \psi
\end{gathered}
$$


Set 2 is defined by

$$
\begin{gathered}
q \cos \chi \mathrm{M}_{5}+m q \sin \chi\left(\mathrm{M}_{+}+\mathrm{M}_{-}\right)=\bar{\Delta}_{3}, \\
Q_{5} \mathrm{M}_{5}+2 d_{5} m q^{2} \sin \chi \cos \chi\left(\mathrm{M}_{+}+\mathrm{M}_{-}\right)=\bar{\Sigma}_{1} /(\mu p), \\
q^{2} \sin \chi \cos \chi \mathrm{M}_{5}+\left(m q^{2} \sin ^{2} \chi-Q_{+}\right) \mathrm{M}_{+}+\left(m q^{2} \sin ^{2} \chi-Q_{-}\right) \mathrm{M}_{-}=\bar{\Sigma}_{2} /(\mu p), \\
\mathrm{M}_{5}=U_{5}^{(+)}-U_{5}^{(-)}, \quad \mathrm{M}_{ \pm}=A_{ \pm}\left(U_{ \pm}^{(+)}-U_{ \pm}^{(-)}\right) .
\end{gathered}
$$

Solution of (B.1a)-(B.1c) gives

$$
\begin{aligned}
\mathrm{P}_{5} & =\frac{1}{C_{0}}\left(q \cos \chi \frac{\bar{\Sigma}_{3}}{\mu p}+Q_{5} \bar{\Delta}_{1}+q^{2} \sin \chi \cos \chi \bar{\Delta}_{2}\right), \\
\mathrm{P}_{+} & =\frac{Q_{-}}{m C_{0} \Omega_{+} \Omega_{-} q \sin \chi}\left(\frac{\bar{\Sigma}_{3}}{\mu p}+2 d_{5} q \cos \chi \bar{\Delta}_{1}+q \sin \chi \bar{\Delta}_{2}\right)-\frac{\bar{\Delta}_{2}}{\Omega_{+} \Omega_{-}}, \\
\mathrm{P}_{-} & =\frac{-Q_{+}}{m C_{0} \Omega_{-} \Omega_{+} q \sin \chi}\left(\frac{\bar{\Sigma}_{3}}{\mu p}+2 d_{5} q \cos \chi \bar{\Delta}_{1}+q \sin \chi \bar{\Delta}_{2}\right)+\frac{\bar{\Delta}_{2}}{\Omega_{-} \Omega_{+}} .
\end{aligned}
$$

The results for (B.2a)-(B.2c) are

$$
\begin{aligned}
& \mathrm{M}_{5}=\frac{1}{C_{0}}\left(\frac{\bar{\Sigma}_{1}}{\mu p}-2 d_{5} q \cos \chi \bar{\Delta}_{3}\right) \\
& \mathrm{M}_{+}=\frac{Q_{-}}{m C_{0} \Omega_{+} \Omega_{-} q \sin \chi}\left(Q_{5} \bar{\Delta}_{3}-q \cos \chi \frac{\bar{\Sigma}_{1}}{\mu p}\right)+\frac{1}{\Omega_{+} \Omega_{-}}\left(\frac{\bar{\Sigma}_{2}}{\mu p}-q \cos \chi \bar{\Delta}_{3}\right), \\
& \mathrm{M}_{-}=\frac{-Q_{+}}{m C_{0} \Omega_{-} \Omega_{+} q \sin \chi}\left(Q_{5} \bar{\Delta}_{3}-q \cos \chi \frac{\bar{\Sigma}_{1}}{\mu p}\right)+\frac{1}{\Omega_{-} \Omega_{+}}\left(\frac{\bar{\Sigma}_{2}}{\mu p}-q \cos \chi \bar{\Delta}_{3}\right) .
\end{aligned}
$$

In light of (B.1d) and (B.2d),

$$
U_{5}^{( \pm)}=\frac{1}{2}\left(\frac{\mathrm{P}_{5}}{A_{5}}( \pm) \mathrm{M}_{5}\right), \quad U_{ \pm}^{( \pm)}=\frac{1}{2}\left(\mathrm{P}_{ \pm}( \pm) \frac{\mathrm{M}_{ \pm}}{A_{ \pm}}\right)
$$

\section{Appendix $\mathbf{C}$}

$$
\begin{aligned}
S_{1} & =\frac{Q_{5}^{2}}{C_{0}}+\frac{4 d_{5}^{2} A_{5}}{C_{0} \Omega_{+} \Omega_{-}}\left(Q_{-} A_{+}-Q_{+} A_{-}\right) q^{2} \sin \chi \cos \chi \\
S_{12}= & \frac{1}{C_{0}}\left[Q_{5}+\frac{2 d_{5} A_{5}}{\Omega_{+} \Omega_{-}} q^{2} \sin \chi \cos \chi\left(Q_{-} A_{+}-Q_{+} A_{-}\right)\right]+\frac{2 m d_{5}}{\Omega_{+} \Omega_{-}}\left(A_{-}-A_{+}\right) q^{2} \sin \psi \cos \chi \\
S_{2}= & \frac{1}{C_{0}}\left[\cos ^{2} \chi+\frac{2 A_{5}}{\Omega_{+} \Omega_{-}}\left(Q_{-} A_{+}-Q_{+} A_{-}\right)\right] q^{2} \sin ^{2} \chi \\
& +\frac{2 A_{5}}{\Omega_{+} \Omega_{-}}\left[A_{-}\left(2 m q^{2} \sin ^{2} \chi-Q_{-}\right)-A_{+}\left(2 m q^{2} \sin ^{2} \chi-Q_{+}\right)\right]
\end{aligned}
$$




$$
\begin{aligned}
& S_{3}=\frac{1}{C_{0}}\left[4 d_{5}^{2} q^{2} \cos ^{2} \chi+\frac{Q_{5}^{2}}{\Omega_{+} \Omega_{-} A_{5}}\right.\left.\left(\frac{Q_{-}}{A_{+}}-\frac{Q_{+}}{A_{-}}\right)\right] \\
&+\frac{q^{2} \sin ^{2} \chi}{\Omega_{+} \Omega_{-} A_{5}}\left[\frac{1}{A_{-}}\left(2 m Q_{5}-Q_{-}\right)-\frac{1}{A_{+}}\left(2 m Q_{5}-Q_{+}\right)\right], \\
& T_{1}=\frac{d_{5}}{\Omega_{+} \Omega_{-}}\left[A_{-}\left(Q_{+}-m Q_{5}\right)-A_{+}\left(Q_{-}-m Q_{5}\right)\right] q^{2} \sin \chi \cos \chi \\
& T_{2}=\frac{Q_{5}}{\Omega_{+} \Omega_{-}}\left[A_{-}\left(Q_{-} m q^{2} \sin ^{2} \chi\right)-A_{+}\left(Q_{+}-m q^{2} \sin ^{2} \chi\right)\right] \\
&+\frac{1}{\Omega_{+} \Omega_{-}}\left[A_{-}\left(Q_{+}-m C\right)-A_{+}\left(Q_{-}-m C\right)\right] q^{2} \sin ^{2} \chi
\end{aligned}
$$

\section{Appendix D}

For a Category 1 material with $c_{R}<\dot{c}_{5}<c_{-}$,

$$
\begin{aligned}
& G_{3}^{+}=\exp \left[-\frac{1}{\pi} \int \frac{d u}{u-c} \frac{\Phi_{3}}{1+q(u-c)}\right], \\
& G_{3}^{-}=\exp \left[\frac{1}{\pi} \int \frac{d u}{u+c} \frac{\Phi_{3}}{1-q(u+c)}\right]
\end{aligned}
$$

Integration is over the range $c_{5}<u<c_{+}$, where

$$
\begin{gathered}
c_{5}<u<c_{-}: \Phi_{3}=\tan ^{-1} \frac{a_{-} p_{+}-a_{+} p_{-}}{4 d_{5}^{2} \omega_{+} \omega_{-} a_{+} a_{-} \alpha_{5} \cos ^{2} \chi}, \\
c_{-}<u<c_{+}: \Phi_{3}=\tan ^{-1} \frac{p_{+}}{a_{+}} \frac{\alpha_{-}-4 d_{5}^{2} \omega_{+} \omega_{-} \alpha_{-} \alpha_{5} \cos ^{2} \chi}{p_{ \pm}}=q_{5}^{2} q_{\mp}+\left(q_{ \pm}-2 m q_{5}\right) \sin ^{2} \chi\left(\sin ^{2} \chi-u^{2}\right) \\
\alpha_{-}=\sqrt{-\cos ^{2} \chi-D_{+}^{2} / d_{1}}, \quad \alpha_{5}=\frac{1}{\sqrt{d_{5}}} \sqrt{u^{2} c_{5}^{2}} .
\end{gathered}
$$

Here $\left(a_{5}, q_{5}, a_{ \pm}, q_{ \pm}, D_{+}, \omega_{ \pm}\right)$are functions of $u$ (see $\left.(21)\right)$.

\section{Appendix E}

$$
\begin{gathered}
\chi=0: m_{ \pm}=m_{5}=0, \\
R_{0}=-2 d_{5}\left(1-\frac{d_{5}}{d_{1}}\right), \quad \mathrm{E}_{0}=-2\left(1+\frac{d_{5}}{d_{1}}\right)\left(1-\frac{d_{5}}{8 d_{1}}\right), \\
\chi=\pi / 2: m_{ \pm}=1-\frac{d_{\mp}}{d_{1}}, \quad m_{5}=1-\frac{1}{d_{5}}, \quad \mathrm{~N}_{ \pm}=m-1+d_{\mp}^{2}, \\
R_{2}=-\sqrt{\frac{d_{5}}{d_{2}}} \frac{2 m-2+\Gamma}{\sqrt{\Gamma+2 \sqrt{d_{1} d_{2}}}}
\end{gathered}
$$




$$
\begin{aligned}
& \mathrm{E}_{2}=\frac{\mathrm{T}_{+} \mathrm{N}_{-}^{2}}{\pi d^{2} d_{-}^{2}}\left(1-d_{-}^{2}\right)\left[1+\frac{d_{1}}{m^{2}}\left(1-d_{-}^{2}\right)\right] \\
& +\frac{\mathrm{T}_{-} \mathrm{N}_{+}^{2}}{\pi d^{2} d_{+}^{2}}\left(1-d_{+}^{2}\right)\left[1+\frac{d_{1}}{m^{2}}\left(1-d_{+}^{2}\right)\right]-\frac{2 \mathrm{~T}_{+}^{-}}{\pi d^{2}} \mathrm{~N}_{+} \mathrm{N}_{-}\left(\sqrt{d_{1} / d_{2}}-1\right), \\
& \chi=\pi / 4: m_{ \pm}=\frac{1}{2}\left(1-\frac{d_{\mp}^{2}}{d_{1}}\right), \quad m_{5}=\frac{1}{2}\left(1-\frac{1}{d_{5}}\right), \\
& \mathrm{N}_{ \pm}=\frac{1}{2}\left[m-\left(1+2 d_{5}\right)\left(1-d_{\mp}^{2}\right)\right], \quad a_{ \pm}^{0}=\frac{1}{\sqrt{2}} \sqrt{1+d_{\mp}^{2} / d_{1}}, \\
& R_{4}=4 d_{5}^{2}-\frac{\sqrt{d_{5}}}{a_{-}^{0} d \sqrt{2} \sqrt{1+d_{5}}}\left[\left(1-d_{-}^{2}\right)\left(1+2 d_{5}\right)^{2}+1-d_{+}^{2}-2 m\left(1+2 d_{5}\right)\right] \\
& +\frac{\sqrt{d_{5}}}{a_{+}^{0} d \sqrt{2} \sqrt{1+d_{5}}}\left[\left(1-d_{+}^{2}\right)\left(1+2 d_{5}\right)^{2}+1-d_{-}^{2}-2 m\left(1+2 d_{5}\right)\right], \\
& \mathrm{E}_{4}=\frac{2 \mathrm{~T}_{+} \mathrm{N}_{-}^{2}}{\pi\left(a_{+}^{0} d\right)^{2} d_{1}}\left(1-d_{-}^{2}\right)\left[1+\frac{d_{1}}{m^{2}}\left(1-d_{-}^{2}\right)\right]+\frac{2 \mathrm{~T}_{-} \mathrm{N}_{+}^{2}}{\pi\left(a_{-}^{0} d\right)^{2} d_{1}}\left(1-d_{+}^{2}\right)\left[1+\frac{d_{1}}{m^{2}}\left(1-d_{+}^{2}\right)\right] \\
& -\frac{4 \mathrm{~T}_{+}^{-}}{\pi d^{2}} \mathrm{~N}_{+} \mathrm{N}_{-}\left(\frac{\sqrt{d_{1}}}{c_{+} c_{-}}-1\right)+\frac{2 \mathrm{~T}_{5}}{\pi} d_{5}+\frac{4 \sqrt{d_{5}}}{\pi d}\left[\mathrm{~T}_{5}^{-} \mathrm{N}_{+}\left(\frac{c_{5}}{a_{-}^{0}}-\sqrt{d_{5}}\right)-\mathrm{T}_{5}^{+} \mathrm{N}_{-}\left(\frac{c_{5}}{a_{+}^{0}}-\sqrt{d_{5}}\right)\right] \text {. }
\end{aligned}
$$

\section{References}

[Abramowitz and Stegun 1972] M. Abramowitz and I. A. Stegun (editors), Handbook of mathematical functions, Dover, New York, 1972.

[Achenbach 1973] J. D. Achenbach, Wave propagation in elastic solids, North-Holland, Amsterdam, 1973.

[Achenbach and Brock 1973] J. D. Achenbach and L. M. Brock, "On quasistatic and dynamic fracture", pp. 529-541 in Proceedings of an international conference on Dynamic Crack Propagation, 1973.

[Barber 1992] J. R. Barber, Elasticity: solid mechanics and its applications, Kluwer, Dordrecht, 1992.

[Brock 2012] L. M. Brock, "Two cases of rapid contact on an elastic half-plane: the sliding ellipsoid, rolling sphere", J. Mech. Mater. Struct. 7:5 (2012), 469-483.

[Brock 2013] L. M. Brock, "Rapid sliding contact in three dimensions by dissimilar elastic bodies: effects of sliding speed and transverse isotropy", J. Mech. Mater: Struct. 8:8 (2013), 461-477.

[Brock 2017a] L. M. Brock, "Transient analysis of fracture initiation in a coupled thermoelastic solid", J. Mech. Mater. Struct. 12:5 (2017), 667-688.

[Brock 2017b] L. M. Brock, "Transient growth of a planar crack in three dimensions: mixed mode", J. Mech. Mater. Struct. 12:3 (2017), 313-328.

[Brock and Achenbach 1973] L. M. Brock and J. D. Achenbach, "Extension of an interface flaw under the influence of transient waves", Int. J. Solids Struct. 9:1 (1973), 53-68.

[de Boer et al. 1988] F. R. de Boer, R. Boom, W. C. M. Mattens, A. R. Miedema, and A. K. Niessen, Cohesion in metals, North-Holland, Amsterdam, 1988.

[Freund 1972] L. B. Freund, "Energy flux into the tip of an extending crack in an elastic solid", J. Elasticity 2:4 (1972), 341-349.

[Freund 1990] L. B. Freund, Dynamic fracture mechanics, Cambridge University Press, 1990.

[Gdoutos 2005] E. E. Gdoutos, Fracture mechanics: an introduction, Solid mechanics and its applications 123, Springer, New York, 2005. 
[Jones 1999] R. M. Jones, Mechanics of composite materials, 2nd ed., Taylor and Francis, New York, 1999.

[Malvern 1969] L. S. Malvern, Introduction to the mechanics of continuous media, Prentice-Hall, Englewood Cliffs, NJ, 1969.

[Morse and Feshbach 1953] P. F. Morse and H. Feshbach, Methods of theoretical physics, McGraw-Hill, New York, 1953.

[Payton 1983] R. G. Payton, Elastic wave propagation in transversely isotropic solids, Martinus Nijhoff, The Hague, 1983.

[Scott and Miklowitz 1967] R. A. Scott and J. Miklowitz, "Transient elastic waves in anisotropic plates", J. Appl. Mech. (ASME) 34:1 (1967), 104-110.

[Skriver and Rosengaard 1992] H. L. Skriver and N. M. Rosengaard, "Surface energy and work functions of elemental metals", Phys. Rev. B 46:11 (1992), 7157-7168.

[Sneddon 1972] I. N. Sneddon, The use of integral transforms, McGraw-Hill, New York, 1972.

Received 6 Oct 2017. Accepted 13 Feb 2018.

LOUIS M. BROCK: louis .brock@uky. edu

Department of Mechanical Engineering, University of Kentucky, Lexington, KY, United States 